\title{
Retrospective analysis of head and neck masses diagnosed by cytology in a tertiary care centre
}

\author{
Subitha $K^{1}$, Sheeja $S^{2}$, Renu Thambi ${ }^{3}$, Jayalakshmy PL ${ }^{4}$, Lillykutty Pothen ${ }^{5}$, Letha $V^{6}$ \\ ${ }^{1}$ Dr. Subitha K, Assistant Professor, ${ }^{2}$ Dr. Sheeja. S, Assistant Professor, ${ }^{3}$ Dr. Renu Thambi, Assistant Professor, \\ ${ }^{4}$ Dr. Jayalakshmy P.L, Assistant Professor, ${ }^{5}$ Dr. Lillykutty Pothen, Additional Professor, ${ }^{6}$ Dr. Letha.V, Additional \\ Professor; All authors are attached with Department of Pathology, Goverment Medical College, Kottayam (Dist), Kerala, \\ India.
}

Address for Correspondence: Dr. Subitha. K, Assistant Professor, Department of Pathology, Goverment Medical College, Kottayam, Gandhinagar- PO, Kerala. Email: subitha@rediffmail.com

\begin{abstract}
Introduction: Fine Needle Biopsy of head and neck masses as a minimally invasive technique is particularly suitable in this sensitive area where an incisional biopsy can cause problems. A cytological diagnosis of a non-neoplastic lesion or confirming suspected metastatic or recurrent tumor can obviate the need for surgery. FNAC can effectively distinguish between benign and malignant cystic lesions of the head and neck. Aim: The aim of this study is to categorise the various head and neck lesions diagnosed by cytology and to assess the diagnostic utility of FNAC. Materials and Methods: All the cases of head and neck masses diagnosed by cytology during the period of one year were analysed. Age and sex distribution was noted. Lesions were mainly categorised into lesions of thyroid, salivary gland and lymph node. Each group were then subcategorised into various groups depending upon the neoplastic and nonneoplastic nature. In some cases histopathologic correlation was done. Results: Thyroid lesions formed $61.5 \%$ (475) of the total number of head and neck masses. 99\% of the cases were benign lesions. Lymphadenopathies formed 34\% (262) of the total number of head and neck masses. Most common diagnoses were reactive lymphadenitis (43.5\%) followed by granulomatous lymphadenitis (12.5\%). Salivary gland lesions formed $4.5 \%$ (35) of the total number of head and neck masses. Sialadenitis was the most common among the benign lesions (40\%) and pleomorphic adenoma was commonest among neoplasms (37\%). Conclusion: FNAC is a reliable, safe, cost-effective and minimally invasive procedure. FNAC can be advised as a primary diagnostic tool.
\end{abstract}

Keywords- Head and neck masses, FNAC

\section{Introduction}

The proximity of tissues of various types and the wide range of primary and metastatic neoplasms are responsible for the head and neck lesions being among the most interesting and challenging in FNAC diagnosis [1]. FNAC, as a minimally invasive technique, is particularly suitable in this sensitive area where an incisional biopsy can cause problems [2]. A cytological diagnosis of a non-neoplastic lesion or confirming suspected metastatic or recurrent tumor can obviate the need for surgery [2]. In other cases, categorization of disease to guide clinical management including further investigation, appropriate referral or rational planning

Manuscript received: $24^{\text {th }}$ October 2016

Reviewed: $5^{\text {th }}$ November 2016

Author Corrected: $15^{\text {th }}$ November 2016

Accepted for Publication: $27^{\text {th }}$ November 2016 of surgery can be offered. A type-specific cytological diagnosis is often possible but may require special experience and the use of ancillary laboratory techniques.

The investigation of suspected local recurrence or nodal metastasis of previously diagnosed cancer is a common indication for FNB in the head and neck [3]. It is of considerable clinical value in the management of these patients since therapeutic decisions can be made without delay and without the need for further diagnostic surgery. FNAC can effectively distinguish between benign and malignant cystic lesions of the head and neck [4]. It is usually not difficult to distinguish between tumor recurrence, on the one hand, and 


\section{Research Article}

inflammation or scarring, on the other. However, it may be difficult to locate a small recurrence in an area of post-radiation edema and fibrosis and to obtain a representative sample.

The potential for cytological diagnosis of all kinds of lesions in the head and neck has been confirmed by numerous case reports and small series of cases, but relatively few large series of specific entities have been analyzed statistically. In any case, even if a definitive, type-specific diagnosis is not possible, FNB can provide cytological categorization of the disease process with a list of differential diagnoses to guide further investigations.

Fine-needle aspiration (FNA) is a well-established procedure for the evaluation of lymphadenopathy, which occurs in a wide spectrum of diseases including reactive conditions, infections, and primary and metastatic malignancies [5]. FNAC is the simplest, least invasive and least costly method which will help to distinguish between cases of lymphadenopathy with a high or a low level of suspicion of significant disease. An immediate decision can be made whether to simply observe the node, to recommend a course of antibiotics, or to refer the patient to a specialist for further investigations.

Reactive lymphadenopathy is likely to resolve spontaneously in due course. Since the great majority of lymphadenopathy cases seen at the primary level are reactive, this approach has obvious practical, economical and psychological advantages. In case of reactive hyperplasia, surgical excision is not indicated, unless the subsequent course is atypical or there is significant discrepancy with the clinical or imaging findings. Follow-up is still recommended, since there is a small but significant risk that lymphoma or other malignancy could be missed or under called by FNA. A specific diagnosis can be made in some cases in this category, for example of an infectious process, but most often the etiology remains obscure. If appropriate, and depending on the clinical presentation, part of the biopsy material may be used for microbiological investigation, which can be supplemented by serology. Literature has established the role of FNA in the evaluation of primary and recurrent non-Hodgkin's lymphoma (NHL), particularly when combined with immunophenotypic and molecular genetic studies.

Preoperative distinction of benign lesions of thyroid is of paramount importance to avoid unnecessary surgery.
FNA is a first-line diagnostic test in the preoperative evaluation of thyroid lesions because of its simplicity, diagnostic accuracy and cost effectiveness.

FNA has been shown to be able to categorise many benign and malignant lesions and thereby guide therapeutic protocols. It is also useful in the diagnosis and monitoring of autoimmune thyroid lesions, especially in clinically equivocal cases and cases where biochemical and immunological parameters are normal or marginally abnormal. [3] US-guided FNA of thyroid is useful, especially in cystic and multinodular lesions harbouring malignancy.

Combination of core needle biopsy with FNA increases diagnostic accuracy but the problem of distinguishing benign and malignant FNs remains. In general, safety and ease of use of FNA outweigh the slight increase in accuracy achieved by core needle biopsy.

A variety of lesions can occur in salivary gland- benign and malignant neoplasms, tumor like lesions, cystic lesions, and inflammatory lesions etc. A definitive type specific diagnosis is not possible by FNA. The exact nature of the lesion can be diagnosed only by microscopic evaluation. Salivary gland tumors are generally not subjected to incisional or core needle biopsy because of the possible risk of causing a fistula or disruption of the capsule with seeding of tumor cells and subsequent recurrence. Fine-needle aspiration (FNA), poses a negligible risk of complications and so FNAC can be combined with clinical and radiological findings is to provide a preliminary assessment on which management decisions can be based.

Head and neck masses are the commonest lesions in patients who attend the cytology department. The ability of FNAC to diagnose all kinds of neoplastic and non neoplastic lesions in the head and neck has been confirmed by numerous case reports and studies. This retrospective study was designed to review the diversity of lesions that can occur in the head and neck region and to analyse neoplastic and non-neoplastic lesions of head and neck region diagnosed by cytology. The utility and diagnostic accuracy of FNAC was also assessed.

\section{Materials and Methods}

All the cases of head and neck masses diagnosed by cytology during the period of one year in the cytology department of Government Medical College Kottayam were analysed. 


\section{Research Article}

\section{Study Design- Retrospective Cohort study}

Inclusion Criteria- All cases of head and neck masses reported in cytology department of Govt. Medical College, Kottayam.

Exclusion Criteria- Those cases in which material was not adequate were excluded.

Fine needle aspiration of neoplastic and non neoplastic lesions in head and neck region are done after obtaining clinical data of the desired subjects and taking consent from the patients. The patient is explained about the procedure including limitations and complications. Patient is made to sit in upright position or lie in supine position and after taking proper aseptic precautions, the procedure is carried out. 10 or $20 \mathrm{ml}$ syringe along with 23 to 25 Gauge needles are used for aspiration.
The smears are either air dried or wet fixed and then routinely stained by Giemsa and Papanicolaou stain wherever required. Descriptive statistics was used for analysis and interpretation of data. All the cases of head and neck masses diagnosed by cytology during the period of one year were analysed. Age and sex distribution were noted.

Lesions were mainly categorised into lesions of thyroid, salivary gland and lymph node. Each group was then subcategorised into various groups depending upon the neoplastic and nonneoplastic nature. In cases diagnosed by cytology, excision biopsy was done in many of the neoplastic lesions. In those cases histopathologic correlation was also done. Diagnostic tests were applied to find the sensitivity, specificity and accuracy of the test.

\section{Results}

A total of 772 FNAC from head and neck lesions were performed, which included 475 cases of thyroid lesions (61.5\%), 262 cases from lymph node (34\%) and 35 cases of salivary gland lesions (4.5\%). The patterns of various lesions are displayed in Tables 1, 2 and 3 respectively.

Thyroid lesions- The thyroid lesions are categorised according to the Bethesda classification. 89\% (425) of the cases were females and $11 \%$ (50) were males.Among the total number of 475 cases, $99 \%$ of the cases were benign lesions and only $1 \%$ was malignant. The youngest patient was a 5 year old diagnosed with multinodular goitre. The oldest patient was an 88 year old male with multinodular goitre.

Table-1: Thyroid lesions.

\begin{tabular}{|c|c|c|c|}
\hline Bethesda Category & Cytologic Diagnosis & No. of Cases & \% percentage \\
\hline II )Benign Follicular Nodule & Mult inodular goitre & 339 & 71 \\
\hline & Hyperplastic nodule & 28 & 6 \\
\hline & Cystic lesions & 3 & 1 \\
\hline & Lymphocytic thyroiditis & 76 & 4 \\
\hline & Hashimoto's thyroiditis & 21 & 1 \\
\hline IV)Follicular neoplasm & Granulomatous thyroiditis & 03 & 1 \\
\hline VI) Malignant & Papillary Carcinoma thyroid & 03 & \\
\hline & Anaplastic Carcinoma & 01 & \\
\hline
\end{tabular}

HP correlation-Out of 475 cases of thyroid lesions histopathological correlation was possible in 104 cases only. There was positive correlation in 93 cases and 11 cases showed negative correlation.

Lesions of the Lymph node- There were a total of 262 cases of which 196 cases were benign lesions and 66 cases were malignant. $69 \%$ (180) of the cases were males and $31 \%$ (82) were females.

The youngest patient of the series was a one year old child diagnosed with a reactive lymphadenitis and the oldest patient was a 90 year old male with metastatic squamous cell carcinoma.

HP correlation- Histopathology correlation was possible in 21 cases of which 18 cases showed positive correlation and 3 cases showed negative correlation. 
Research Article

Table-2: Lesions of Lymph node.

\begin{tabular}{|c|c|c|c|}
\hline S. No. & Cytologic Diagnosis & No. of Cases & \% percentage \\
\hline Benign (196) & Reactive lymphadenitis & 114 & 43.5 \\
\hline & Granulomatous lymphadenitis & 33 & 12.5 \\
\hline & Suppurative lymphadenitis & 30 & 11 \\
\hline & Tuberculous lymphadenitis & 19 & 7 \\
\hline Malignant (66) & Metastasis from Squamous cell carcinoma & 27 & 10 \\
\hline & Metastasis from Adenocarcinoma & 16 & 5 \\
\hline & Metastasis from poorly differentiated carcinoma & 14 & 1 \\
\hline & Metastasis from small cell carcinoma & 1 & 1 \\
\hline & Metastais from Papillary carcinoma thyroid & 1 & 3 \\
\hline
\end{tabular}

Salivary gland lesions- There were 35 cases of salivary gland lesions of which 34 cases were benign and only one case of malignancy.51\% (18) of the cases were males and 49\% (17) were females. The youngest patient was a 11 year old girl with sialadenitis and the oldest patient was a 71 year old male with pleomorphic adenoma.

Table-3: Salivary gland lesions.

\begin{tabular}{|c|c|c|c|}
\hline & Cytologic Diagnosis & No. of Cases & \% Percentage \\
\hline Benign lesions. & Sialadenitis & 15 & 40 \\
\hline & Cystic lesion & 03 & 08 \\
\hline Benign neoplasms & Spindle cell lesion & 01 & 37 \\
\hline & Pleomorphic adenoma & 13 & 08 \\
\hline Malignant neoplasms & Warthin's tumor & 03 & 03 \\
\hline Total No of Cases & Mucoepidermoid carcinoma & 01 & \\
\hline
\end{tabular}

HP correlation- Out of 35 cases histopathological correlation was available in only 6 cases.5 cases showed positive correlation and 1 case showed negative correlation.

Table-4: Thyroid Cases- Histopathology Correlation.

\begin{tabular}{|c|c|c|c|}
\hline & Histopathology & & Total \\
\hline Cytology & Malignant & 0 & 2 \\
\hline Malignant & 2 & 91 & 102 \\
\hline Benign & 11 & $\mathbf{9 1}$ & $\mathbf{1 0 4}$ \\
\hline Total & $\mathbf{1 3}$ & Benign \\
\hline
\end{tabular}

Sensitivity: $15.38 \%$, Specificity: $100 \%$, Accuracy: $89.42 \%$

Positive Predictive value: $100 \%$, Negative predictive value: $89.22 \%$,

Likelihood Ratio of Negative test: .85

Table-5: Lymph Node lesions-Histopathology Correlation.

\begin{tabular}{|c|c|c|c|}
\hline & Histopathology & & \\
\hline Cytology & Malignant & Benign & 2 \\
\hline Malignant & 2 & 0 & 19 \\
\hline Benign & 3 & 16 & $\mathbf{2 1}$ \\
\hline Total & $\mathbf{5}$ & $\mathbf{1 6}$ & Total \\
\hline
\end{tabular}

Sensitivity: 40\%, Specificity: 100\%, Accuracy: $85.7 \%$

Positive predictive value: $100 \%$, Negative predictive value: $84.2 \%$,

Likelihood ratio of Negative test: 0.6 
Research Article

Table-6: Salivary gland lesions-Histopathology correlation.

\begin{tabular}{|c|c|c|c|}
\hline & Histopathology & & Total \\
\hline Cytology test & Malignant & Benign & 0 \\
\hline Malignant & 0 & 0 & 6 \\
\hline Benign & 1 & 5 & $\mathbf{6}$ \\
\hline Total & $\mathbf{1}$ & $\mathbf{5}$ & \\
\hline
\end{tabular}

Sensitivity: 0, Specificity: $100 \%$, Accuracy: $83.3 \%$.

Negative predictive value: .83 , Likelihood ratio for negative test: 1

\section{Discussion}

All the cases of head and neck masses diagnosed by cytology during a period of one year were analysed. A total of 772 cases were studied. We obtained different data like age and sex distribution and the lesions were subcategorised into various groups. The results achieved in the present study were compared with different studies.

Lesions of Thyroid- Thyroid lesions formed 61.5\% (475) of the total number of head and neck masses. $99 \%$ of the cases were benign lesions of which majority of the cases were benign follicular nodule-Multinodular goitre. This was similar to a study by Naila Tariq [6] which showed $56.9 \%$ of multinodular goitre. In another study by Farzana Shahid et al benign nodular goitre formed $52.4 \%$ of the cases. [2] A diagnosis of hyperplastic nodule was given in cases where there was overlapping features of a nodular goitre and a macro follicular adenoma.

One case was diagnosed as follicular neoplasm with the presence of microfollicles, increased cellularity and scanty colloid [3]. There were only three case of papillary carcinoma and one case of anaplastic carcinoma. Malignant neoplasms formed only $1 \%$ of the total cases. This was similar to other studies by Naila et al $(1.53 \%)$ and Farzana et al $(3.2 \%)[2,6]$.

There was positive histopathology correlation in 93 cases of thyroid lesions and 11 cases showed negative correlation. (Table 4) Among the cases which showed positive correlation 91 cases were benign. This shows that FNAC is highly specific for diagnosing benign conditions of the thyroid. Our study had a sensitivity $15.38 \%$, Specificity- $100 \%$, Accuracy- $89.42 \%$, Positive Predictive value: $100 \%$, Negative predictive value: $89.22 \%$, Likelihood Ratio of Negative test: .85. This was comparable to another study where FNAC had a ensitivity of $52.6 \%$, specificity of $86.6 \%$, NPV of
$90.4 \%$, PPV of $38.4 \%$ and accuracy of $79.1 \%$ [9]. Six cytologically diagnosed cases of colloid goitre turned out to be papillary carcinoma on histopathology. It may be because the nuclear features of papillary carcinoma were missed. False negative reports usually occur in cases of cystic papillary carcinoma and follicular variant of papillary carcinoma. Cystic change can occur in $25 \%$ cases of papillary carcinoma thyroid. When cystic change occurs in solitary nodules of thyroid, always rule out the possibility of a papillary carcinoma.

Abundant colloid may be present in cystic and macro follicular variants of papillary carcinoma. The amount of colloid present is not a reliable indicator whether the lesion is benign or malignant.Also presence of papillary structures does not always mean papillary carcinoma. Multiple aspirations are advised in a thyroid swelling to get a more representative sample. Look for the typical nuclear features. In cases of papillary microcarcinoma lesion may be missed and may be interpreted as nodular goitre. To avoid this ultrasound guided aspiration is advised in cases of small lesions.

Lesions of Lymph node- Lymphadenopathies formed $34 \%$ (262) of the total number of head and neck masses. Most common diagnoses were reactive lymphadenitis (43.5\%) followed by granulomatous lymphadenitis. $(12.5 \%)$. A definite diagnosis of tuberculous lymphadenitis was given in only 19 cases. In a study by El-Hag et al reactive lymphadenitis was the commonest cause of neck masses [10]. But in study by Farzana etal granulomatous lymphadenitis was the commonest among lymphadenopathies [2]. Tuberculous lymphadenitis was the commonest cytological diagnosis in a study by Saira Fatima [8].

A study by Amatya etal showed that FNAC of head and neck lesions is a useful investigation which provides an accurate and cost-effective preoperative diagnosis of 


\section{Research Article}

head and neck masses [7]. The sensitivity of FNAC in this study was $77 \%$ and specificity was $98 \%$. The overall diagnostic accuracy in detecting malignant tumors was $93 \%$ with positive predictive and negative predictive values of $91 \%$ and $93 \%$ respectively. In a study by Fatima etal FNAC was helpful in establishing the diagnosis in approximately $98 \%$ of the cases of lymphadenopathy [8].

Histopathology correlation was available in 21 cases. 18 cases showed positive correlation and 3 cases showed a false negative diagnoses. (Table-5) Our study had a sensitivity $40 \%$, Specificity-100\%, Accuracy- $85.7 \%$.

Positive Predictive value: $100 \%$, Negative predictive value: $84.2 \%$, Likelihood Ratio of Negative test: 0.6. 2 cases of Hodgkin lymphoma was diagnosed as granulomatous lymphadenitis. Clusters of epithelioid cells are sometimes seen in smears of Hodgkin lymphoma and in some NHL.

One must therefore look carefully for abnormal lymphoid cells and for nonlymphoid cells in smears containing epithelioid histiocytes [2]. 1 case of suppurative lymphadenitis diagnosed by cytology came out to be tuberculous lymphadenitis in histopathology.

Smears from a tuberculous lymph node may sometimes show only polymorphs and necrotic debris without histiocytes, particularly in immunocompromised patients. Acid-fast bacilli should be looked for both in direct smears and in culture from the aspirate in clinically suspicious cases of tuberculosis.

Lesions of Salivary Gland- Salivary gland lesions formed $4.5 \%$ (35) of the total number of head and neck masses.Sialadenitis was the most common among the benign lesions (40\%) and pleomorphic adenoma was commonest among neoplasms (37\%) In a study by Farzana etal and Setal etal benign neoplasms were commonest followed by inflammatory lesions [2,11].

Out of 35 cases histopathological correlation was available in only 6 cases. 5 cases of Pleomorphic adenoma showed positive correlation and 1 case showed negative correlation (Table 6). Our study had a sensitivity $0 \%$, Specificity - 100\%, Accuracy- $83.3 \%$, Negative predictive value: $84.2 \%$, Likelihood Ratio of Negative test: 1 . A case of mucoepidermoid carcinoma was diagnosed as pleomorphic adenoma in cytology. Review of these smears showed the presence of epithelial clusters and myxoid material which was misinterpreted as chondromyxoid material of pleomorphic adenoma.

\section{Conclusion}

Majority of the thyroid swellings are benign lesions where surgical management is not required. So FNAC of the thyroid can be used as a diagnostic test to distinguish thyroid nodules that have a higher risk of malignancy and which require surgical excision.

FNAC of lymph nodes is a reliable and cost effective method for investigating the nature of the lesions, whether infectious, reactive or neoplastic processes. Non-neoplastic conditions of the salivary gland that simulate tumour are cystic, sialadenitis, granulomatous disease and benign lympho-epithelial lesions. Most of disorders require medical management or minimal surgical intervention, such as cyst aspiration. Diagnosis by FNAC would clearly reduce the amount of surgery. FNAC is a reliable, safe, cost-effective and minimally invasive procedure. Benign lesions can be diagnosed and malignancies can be confirmed and initiate proper management. FNAC can be advised as a primary diagnostic tool.

Funding: Nil, Conflict of interest: None initiated, Permission from IRB: Yes

\section{References}

1. Sharma R, Mathur DR. Fine needle aspiration cytology (FNAC) of palpable lesions of head and neck region. International Journal of Current Research and Review. 2012 Nov 15;4(22):74.

2. Shahid F, Mirza T, Mustafa S, Sabahat S, Sharafat S. An experiential status of fine needle aspiration cytology of head and neck lesions in a tertiary care scenario. Journal of Basic \& Applied Sciences. 2010 Dec 1;6(2).

3. Orell SR, Sterrett GF, Whitaker D. Fine needle aspiration cytology. Churchill Livingstone; 2005 Jul 8.

4. Gray W, Kocjan G. Diagnostic Cytopathology: Expert Consult: Online and Print. Elsevier Health Sciences; 2010 May 24.

5. Bibbo M, Wilbur D. Comprehensive cytopathology. Elsevier Health Sciences; 2014 Sep 5.

6. Tariq N, Sadiq S, Kehar S, Shafiq M. Fine needle aspiration cytology of head and neck lesions-an 


\section{Research Article}

experience at the JINNAH post graduate medical centre, Karachi. Pak J Otolaryngol. 2007;23:63-5.

7. Amatya BB, Joshi AR, Singh SK, Panth R, Basnet RB. A study of fine needle aspiration cytology of head and neck masses and their corroboration by histopathology. Post graduate medical journal of national academy of medical sciences. 2009;6(2).

8. Fatima S, Arshad S, Ahmed Z, Hasan SH. Spectrum of cytological findings in patients with neck lymphadenopathy-experience in a tertiary care hospital in Pakistan. Asian Pac J Cancer Prev. 2011 Jan $1 ; 12(7): 1873-5$.
9. Mundasad B, Mcallister I, Carson J, Pyper PC. Accuracy of fine needle aspiration cytology in diagnosis of thyroid swellings. Internet $\mathbf{J}$ Endocrinol. 2006;2(2):23-5.

10. El-Hag IA, Chiedo LC, AlRayees FA, Kollur SM (2003): FNAC of Head and Neck masses, 7 years experience in a secondary care hospital . acta Cytol., 47 (3): $387-92$

11. Chauhan S, Rathod D, Joshi DS. FNAC of swellings of head and neck region. Indian Journal of applied basic medical sciences. 2011;13(17):1-6.

\section{How to cite this article?}

Subitha K, Sheeja S, Renu Thambi, Jayalakshmy PL, Lillykutty Pothen, Letha V. Retrospective analysis of head and neck masses diagnosed by cytology in a tertiary care centre. Trop J Path Micro 2016;2(3):172-178.doi: 10.17511/jopm.2016.i03.16 